\title{
Detection of Tumor in Mammographic Images by RBF Neural Network and Multi Population Genetic Algorithm
}

\author{
Belgrana Fatima Zohra \\ Department $t$ of Mathematics and Informatics, \\ University of Ain Témouchent, CUAT \\ P.B 284, RP 46000, Ain Témouchent, Algeria
}

\author{
Benamrane Nacéra \\ Department of Informatics, University of Sciences \\ and Technology of Oran "Mohamed BOUDIAF", \\ USTO-MB \\ P.B. 1505, El M'naouer, 31000 Oran, Algeria
}

\begin{abstract}
In this paper, we propose an approach for detection of anomalies present in medical images. The idea is to combine tow metaphors: Neural Networks (NN) and Evolutionary Algorithm (EA) in a hybrid system.

The Radial Basis Function Neural Network (RBF NN) and Multi Population Genetic Algorithm (MPGA) are coupled in one system called neural-evolutionary algorithm.

After applying the growing region algorithm to extract regions, the RBF NN detects the suspect regions. Some of experimental results on mammographic images show the feasibility of the proposed approach.
\end{abstract}

\section{Keywords}

Tumor Detection, Interpretation, RBF NN, MPGA Mammographic Images.

\section{INTRODUCTION}

Breast cancer is a type of cancer originating from breast tissue, most commonly from the inner lining of milk ducts or the lobules that supply the ducts with milk. It has been one of the major causes of death among women since the last decades. If the cancer can be detected early, the options of treatment and the chances of total recovery will increase; in this fact diagnosis of the disease has steadily become more important.

The interpretation of the medicals images plays an important part in the establishment of an early diagnosis and many decisions and therapeutic follow-ups. It represents an important stage in any medical system and consists to give a description of pathological anatomy present in medical images.

Computer-aided diagnostic (CAD) methods have been proposed as one low cost tool to aid radiologists in film interpretation [1], [2]. CAD is notably used to reduce misdiagnosis by providing a second reading and alerting radiologists to locations of suspicious lesions.

Several methods are proposed in order to realize this task; we quote the connectionists approaches (NN), optimizations methods (EA) and the artificial intelligence methodologies which include fuzzy logic and expert systems [3]. Although these metaphors carry out the task of interpretation with some success, they present also disadvantages; to avoid these inconveniences; the hybrid architectures are proposed.
The training of the $\mathrm{NN}$ is carried out on the variety basis of anomalies examples; however the no- interpretability of its layers decreases its capacities of generalization [4]; from where this fuzzy logic intervenes in order to solve this problem thanks to its competence of modelling of unclear and uncertain knowledge [5].

Other hybridizations were realized, which gather the advantages of the NNs, fuzzy logic and genetic algorithms (GA), this parallel neuronal hybrid approach arrive to control NNs black box dimension by specialized neurons introduction [6] [7]. Thus, the final adjustment of the parameters of the fuzzy system is realized by the neuronal training means [8]. The GA is used as initialization of the training step which is doing by the gradient retro propagation algorithm in order to adjust the fuzzy system parameters [9]. In order to determinate the nature of the lesions in medical images; hybridization between fuzzy logic, NNs and expert system was achieved, where the fuzzy-neural approach detects the areas suspect which will be after validate by an expert system [10].

The training of the NN weights is formulated as the error function minimization, such as the average quadratic error between the required output and that obtained by the network [11]. This error function is calculated by the training on a set of image by adjusting the weights of connection repeatedly. Majority of the training algorithms are based on the gradient descent which is often trapped by the local minimum of the error function.

The EA training is used to find, an optimal and global set of connection weight [12]. This approach completely independent of any gradient information, extended on complex spaces, not differentiable, continuous and multimodal was widely applied [13].

In this paper, the idea is to hybrid tow techniques: RBF NN and MPGA in order to detect suspect regions in mammographic images.

This paper is organized as follows literature survey has been presented in section 1 , section 2 presents the proposed approach of which we briefly define the RBF NN and GAMP, section 3 is devoted to the structure of our RBF NN, followed by section 4 in which we will show haw the RBF NN training is done using GAMP. 
Some experimental results are exposed in section 6 , and finally our conclusions and future working lines are given in the last section.

\section{THE HYBRID APPROACH}

\subsection{RBF Neural Network}

A RBF NN is a three-layer supervised feed-forward network that uses a nonlinear transfer function (Gaussian function) for the hidden neurons and a linear transfer function for the output neurons. The Gaussian function is applied to the net input of each neuron to produce a radial function of the distance between each pattern vector and each hidden unit weight vector.

RBF NNs are inherently flexible, and suitable for a variety of problems because of their size and topology; effectively they have been successfully applied to a number of visual processing and analysis problems, we quote time-series and the analysis of 3D structures. They represent very useful tools for medical image analysis [14].

The output of an RBF network is a linear combination of weighted radial basis functions.

$$
\emptyset(x)=\sum_{i=1}^{N} a_{i} \exp \left(-\beta_{j}\right)\left\|x-c_{i}\right\|
$$

Where $N$ is the number of neurons in the hidden layer, $\mathrm{c}_{i}$ is the center of neuron $i$, and $\boldsymbol{a}_{\boldsymbol{i}}$ is weight of the linear output neuron. In the training phase $\boldsymbol{a}_{\boldsymbol{i}}$ and $\boldsymbol{\beta}_{\boldsymbol{j}}$ of each neuron are estimated by a least squares objective function or a regularized objective function. The number of the neuron is set manually, after several tests, our choice was made on 10 neurons.

\subsection{Genetic Algorithm (GA)}

Genetic Algorithm (GA) is a search heuristic that mimic the process of natural selection. This heuristic also called a metaheuristic is routinely used to generate useful solutions to optimization and search problems.

GA has proven to be more efficient than other search algorithm for highly non linear and complex problems [15]. It has no requirement for the continuity in the derivative so virtually any fitness function can be selected for optimizing. Unlike to conventional methods GA use population in which many individuals are selected based on the fitness value in this selection.

In GA the first step is the generation of a random population; the algorithm continues to generate subsequent populations until a halting condition is met. Each individual of population represent a possible solution with different fitness, it is coded by binary string (genotype) which is matched to a set of variable(phenotype); after evaluation step the binary string is decoded using linear mapping. After evaluation of all individuals; the next population is obtained by using genetic operator.

The principal genetic operators used in GA are:

\footnotetext{
- Reproduction,

- Crossover,

- Mutation.
}

GA is used us a basis for building the multi-population GA structure: MPGA.

\subsection{Multi Population Genetic Algorithm}

MPGA is a random population; different from other GA, it uses multiple populations. Each population works on one optimization function corresponding to one task point.

In MPGA the complexity does not increase much as the number of task points increases which represent the main advantage of this algorithm. MPGA is different from GA for multi-optimization function problems [16], since our MPGA solves problems with single complex optimization function; the complexity is divided into multi-optimization functions.

\section{A PROPOSED NEURAL NETWORK}

The choice of the NN was made on the radial basic function neural network; the RBF NN has as characteristic its radial activated functions which give useful answers only in a restricted field of values: the receptive field.

The RBF NNs are much used considering their important advantages in front of the traditional NNs [17].

The evolutionary methods ensure a research in the complete field, progressively with the generations; this research space is refined towards potentially powerful subspaces.

The combination between a total research (MPGA) and a local one (RBF NN) gives an interesting results. It suggests that MPGA find a good configuration of the network weights.

\subsection{Structure of the RBF NN}

The network structure suggested is illustrated in figure 1; it includes an input layer $\left(\mathrm{E}_{1}, \mathrm{E}_{2, \ldots}, \mathrm{E}_{9}\right)$, a hidden and an output layer (S1, S2). The input layer contains 9 neurons; each neuron corresponds to an attribute characterizing an image area, such as the variance, surface, elongation, average gray level, compactness. These attributes are extracted from the image segmentation stage by the region growing approach.

Four other texture attributes are extracted by superposition of the segmented image on the initial one using cooccurrence matrix. These attributes are: homogeneity, contrast, entropy and directivity.

The hidden layer contains 10 neurons, and finally the output layer contains 2 neurons, it represents a linear combination of the output value multiplied by the weights of their connection.

Among the principal parameters to regulate in RBF NN, we quote two of them:

- The number of neurons in the hidden layer.

- The Gaussian centroid position which represents a major problem of the network, to solve this problem, we decide use the k-means algorithm. 


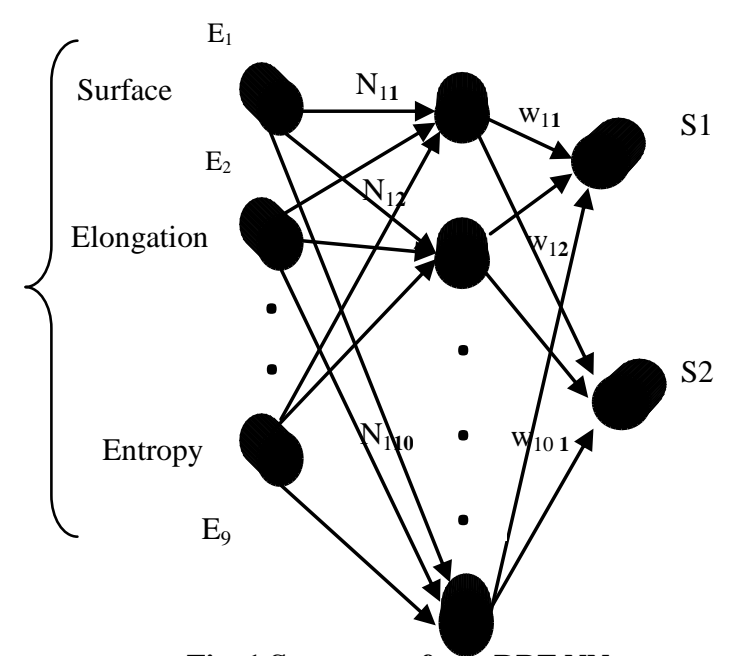

Fig .1 Structure of our RBF NN

Wii: represents the weights of the hidden layer towards the output ones.

Nci: the weights of input layer toward the hidden ones.

\section{TRAINING ALGORITHM OF THE PROPOSED RBF NN}

The training of the RBF NN is supervises at the synaptic weights determination using the MPGA, It is realized according to a training set which is formed by three images containing three anomalies.

Once the neurons weights are adapted, they are stored in a file.

The realization of hybridization consists to define the various modifications necessary to the MPGA to handle the NN. It is necessary in particular to determinate a coding in order to make the NN manipulated by the MPGA.

We will have to define also the various steps of the MPAG and to build operators (crossover, mutation and selection) adapted to the given structure coding types.

The MGAP will see the NN as an individual of a population, each population is formed by chromosomes, and each chromosome represents a juxtaposition of the matrix weights elements.

\subsection{Population Initialization}

In MPGA, each chromosome corresponds to a potential solution. The initial population $\mathbf{Q}$ is composed of a certain number denoted as $\mathbf{n}$ of chromosomes. To explore the genetic diversity, in our algorithm each chromosome is randomly generated.

\subsection{MPGA Immigrants Scheme}

In MPGA, the initial stage is the random generation of a large single population. For each given change interval $I$, the whole population will evolve together for [I/2] generations. Then the single population is split into for small populations of them, we chose four sub populations, one small population will act as the parent population for exploring and the other tree will act as the child populations for exploiting.

Each population evolves according to its own genetic algorithm. We used a binary coding; this choice is justified by the robustness and the problem independence, every individual gene will be coded by an integer of 9 bits.
The random immigrants approach was proposed by Grefenstette [18] which is inspired from the flux of immigration of population individual through generations in nature. The process of replacing some individuals of the current population with random individuals called random immigrants every generation allows to maintain the population diversity level. Concerning the choice of population individuals which should be replaced, there are two strategies: replacing random individuals or replacing the worst ones.

Te ratio of the number of random immigrants to the population size $\boldsymbol{r}_{\boldsymbol{i}}$, is generally set to a small value in order to avoid that random immigrants disrupt the ongoing search progress too much, especially during the static period between two environmental changes

We are inspired from the idea of the Forking genetic algorithms: FGAs, when the parent population continuously searches for new optimum, while a number of child populations try to exploit previously detected promising areas.

\subsection{Fitness Function}

The fitness function must take into account, the average quadratic error $\mathbf{E}$ made by the network in the classification of the examples belonging to the training database:

$$
E=1 / 2 \cdot\left(d_{I}-a_{I}\right)^{2}
$$

With $\mathbf{d}_{\mathbf{I}}$ the desired output value of neuron $\mathbf{I}$ and $\mathrm{a}_{\mathrm{I}}$ its activation function value. In the aim of turning the optimization problem into a maximization of profile function, the fitness function $\mathrm{G}$ of each chromosome is calculated as follows:

$$
G=\left(1-\Sigma^{E} / N\right)^{2}
$$

The selection method used by tow subpopulation is the roulette wheel selection scheme [19], for the tow other we choose the techniques of elitist selection: the $\mathrm{N} / 2$.

The crossover algorithm used is a crossover at one point and crossover at two points. This crossover scheme makes it possible to simultaneously alternate knowledge and rules database.

The mutation operator is a binary traditional mutation scheme with random choice of bits to be muted. We use the offspring replacement scheme which is called Selective Breeding. The fitness value of all children chromosomes is calculated before replacing them; it will be used in the process of replacement, so that a child chromosome replaces another of the initial population only if its fitness value is lower than that of the chromosome parents to replace.

\section{EXPERIMENTAL RESULTS}

To study the performance of the MPGA as an optimization method of the RBF NN and demonstrate the impact of the proposed hybridization, we apply the developed algorithm on 20 mammographic images (see figure 4).

The segmented images present a set of homogeneous areas; each area is characterized by a whole of attributes. The tumor areas are presented to the system during the training stage, other are presented at the test step and generalization stage. The rate of recognition reaches $100 \%$. 

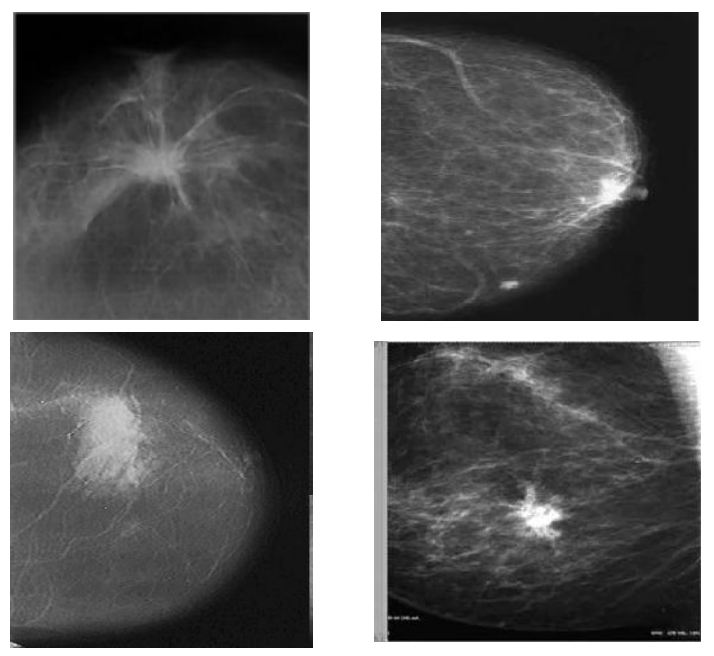

Fig .2 Initial mammographic images
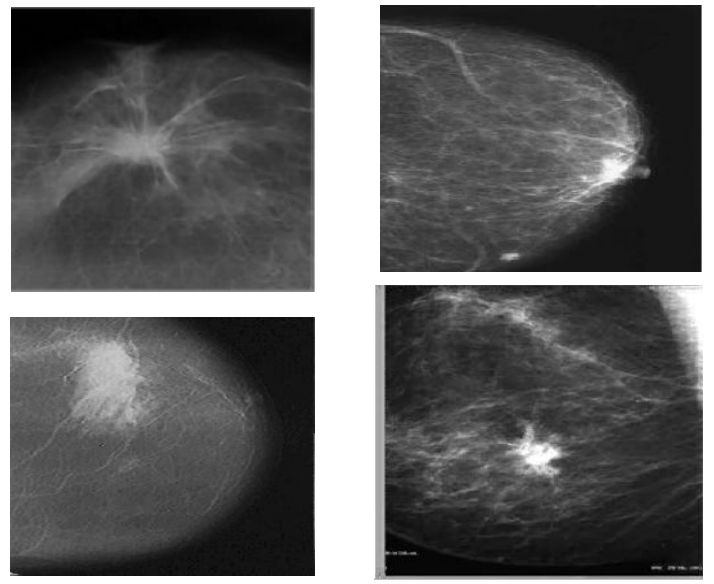

Fig .3 Filtered mammographic images (Median filter)
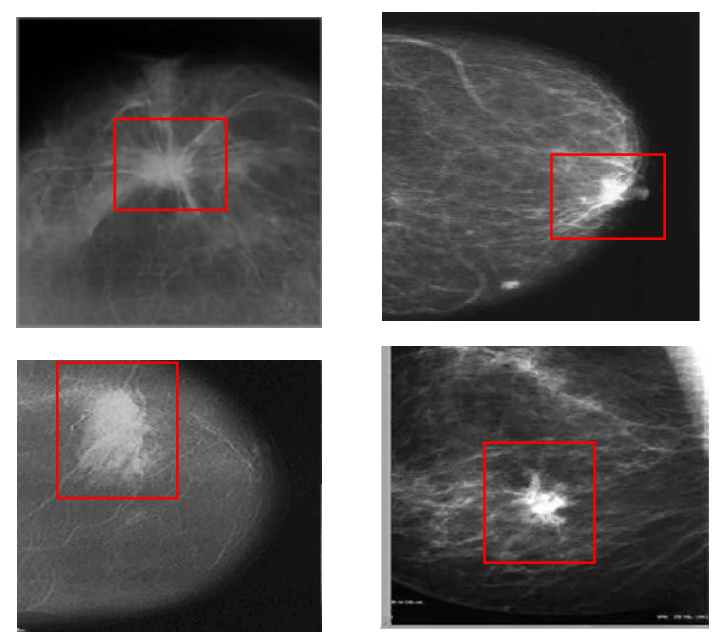

Fig .4 Images with detection of tumor riches $100 \%$.

\section{Conclusion}

In this paper, we have proposed a hybrid approach for tumor detection in medical image. The proposed hybridizing strategy combines into one architecture tow different metaphors: RBF $\mathrm{NN}$ and MPGA. Artificial neural networks mainly arises from their potential ability to perform interesting computational tasks, it had to adopt themselves under unknown situations by the means their training property. The evolutionary algorithms are very powerful; the search for a solution require generally a longer training time, but can give better results than the traditional methods of training like the propagation of the gradient.

The MPGA adds to the RBF NN the possibility of doing global search like an initialization for the traditional neuronal learning algorithm. This hybridizing could deal with several problems caused by the local search training algorithm based on the back propagation of error gradient

The final proposed system could perform the task of tumor detection in medical images.

The proposed approach has been tested on mammographic images. The results of tumors detection and specification were done with very high performances (reach 100\%).

\section{ACKNOWLEDGMENTS}

This work was supported by the National of Agency Research in Health, Project $\mathrm{N}^{\circ}$ 345/ANDRS/2011.

Our thanks to the experts in mammographic clinic; who have contributed with their expertise in medical field and set of mammographic images.

\section{REFERENCES}

[1] Kegelmeyer, W. P. Jr., 1992. Computer detection of stellate lesions in mammograms," in Proc. SPIE Biomed. Image Processing, vol. 1660, pp. 446454.

[2] Dehmeshki, J. 2003. Automated Detection of Nodules in the CT Lung Images using Multi-Modal Genetic Algorithm, Proceedings of the $3^{\text {rd }}$ International Symposium on Image and Signal Processing and Analysis, pp. 393-398.

[3] Benamrane, N., Freville, A. and Nekkahce, R. 2005. Hybrid fuzzy neural network for detection of tumors in medical images", American Journal of Applied Sciences, 2(4) 892-896, ISSN 1546-9239.

[4] M.B. Bouchon, "Fuzzy Logique and its applications ", Edition Addison-Wesley, Paris, 1995.

[5] Nauck, D. 1994. A Fuzzy Perceptron as a Generic Model for Neuro- Fuzzy approaches, Proceedings of Fuzzy Systems and GI-Workshop, Ph.D. Thesis, Department of Computer Science, University of Braunshweig, Germany.

[6] Nauck, D. and Kruse, R. 1997. What are Neuro-Fuzzy Classifiers?, Proceedings of the Seventh International Fuzzy Systems Association World Congress, pp.228233.

[7] Lin, C.T. 1996 Neural Fuzzy System with Fuzzy Supervised Learning, IEEE Trans on System Man, and Cybernetics, Vol. 26(5),

[8] Benamrane, N., Aribi, A. and Kraoula, L. 2006. Fuzzy Neural Networks and Genetic Algorithms for Medical 
Images Interpretation», International Conference on Geometric Modeling and Imaging GMAI06, Londres.

[9] Xin, Y. 1999. Evolving artificial neural networks, Proceeding of the IEEE, Vol 87, No 9.pp.1423-1447.

[10] Laurikkala, J. and Juhola, M. 1999. Comparison of Genetics Algorithms in the Diagnosis Female Urinary Incontinence", Methods of Informatics in medicine, Ph.D. thesis, Department of Computer Science, University of Tampere, Finland.

[11] Sheifer, U. 2001 Multiple layer perceptrons training using genetic algorithms, Proceedings- European Symposium on Artificial Neural Networks, , ISBN 2930307-01-3, pp. 159-164.

[12] Darken, M. 1989 Fast learning in networks of locally tuned processing units, In Neural Computations, vol.1,pp.281-294.
[13] Jiang, J., Trundle, P. and Ren , J. 2010 Medical Image Analysis with Artificial Neural Networks, Digital Media \& Systems Research Institute, University of Bradford, Bradford, BD7 1DP, United Kingdom.

[14] Davidor, Y. 1990 Genetic Algorithm and Robotics, World Scientific: an international publisher, New Jasey.

[15] Goldbag, D.E. 1989 Genetic Algofithmr in Searh, optimizatiom and Mochin Learning, Addison-Wesley publishing Company.

[16] McCulloch, W.S. and Pitts,W. 1943 A logical calculus of the ideas immanent in nervous activity", Bulletin of Mathematical Biophysics, , No 5, pp.115-133.

[17] Grefenstette, J.J. 1992 Genetic algorithms for changing environments. In: Proc. 2nd Int. Conf. on Parallel Problem Solving from Nature, pp. 137-144

[18] Goldberg, D.E. Genetics Algorithms in Search, Optimisation and Machine Learning, Addison Wesley 